\title{
Alterstice
}

Revue internationale de la recherche interculturelle

International Journal of Intercultural Research

Revista International de la Investigacion Intercultural

\section{Échanges en espagnol et en français : intégration linguistique et interculturelle à l'université [synthèse en français]}

\section{Jessica Payeras et Maria Elena Zapata}

Volume 8, numéro 1, 2018

Jumelage interculturel et pédagogie universitaire

URI : https://id.erudit.org/iderudit/1052608ar

DOI : https://doi.org/10.7202/1052608ar

Aller au sommaire du numéro

Éditeur(s)

Alterstice

ISSN

1923-919X (numérique)

Découvrir la revue

Citer cet article

Payeras, J. \& Zapata, M. E. (2018). Échanges en espagnol et en français :

intégration linguistique et interculturelle à l'université [synthèse en français].

Alterstice, 8(1), 59-61. https://doi.org/10.7202/1052608ar 
ARTICLE THÉMATIQUE

\title{
Échanges en espagnol et en français : intégration linguistique et interculturelle à I'université [synthèse en français*]
}

\author{
Jessica Payeras ${ }^{1}$ et Maria Elena Zapata ${ }^{1}$
}

\author{
Rattachement des auteures \\ ${ }^{1}$ Université du Québec à Montréal, Montréal, Canada
}

\section{Correspondance}

payeras.jessica@uqam.ca

\section{Mots clés}

culture, interculturalité, motivation, communication linguistique et interculturelle, rencontre de porteurs de culture, reconnaissance mutuelle

\section{Pour citer cet article}

Payeras, J. et Zapata, M. A. (2018). Échanges en espagnol et en français: intégration linguistique et interculturelle à I'université [synthèse en français]. Alterstice, 8(1), 59-62. [Los intercambios en español y francés: integración lingüística e intercultural en la universidad]

*L'article complet en espagnol est disponible dans le même numéro d'Alterstice, p. 51-58.

Le Québec recevant chaque année environ 50000 migrants dont environ 85 à $90 \%$ dans la région de Montréal, les échanges linguistiques et interculturels constituent un sujet de grand intérêt pour la province, de plus en plus cosmopolite. Selon le recensement 2011 de Statistique Canada, les Latino-Américains représentent 12,9\% de la population montréalaise et ce pourcentage ne cesse d'augmenter.

Cet article décrit une intervention éducative innovante, menée dans le regroupement d'espagnol de l'École de langues à l'Université du Québec à Montréal (UQAM) depuis 2007. Des échanges linguistiques et interculturels ont été mis en place entre les hispanophones (immigrants nouvellement arrivés pour résider dans la province de Québec) inscrits à un programme de langue du ministère de l'Immigration, la Diversité et I'Inclusion (UQAM-MIDI) et les étudiants francophones qui étudient l'espagnol dans le cadre du programme d'espagnol comme langue étrangère (ELE) à I'UQAM.

Le site Internet du programme de français langue étrangère du programme UQAM-MIDI annonce qu' « il s'agit d'un programme dynamique et enrichissant où les cours de français sont dispensés par les professeurs du MIDI et les activités de soutien à la francisation par des animateurs de I'UQAM ». La langue française est au cœur de ce programme, qui porte également sur divers aspects de la société québécoise. 
Le programme d'espagnol de l'UQAM, quant à lui, est un programme enrichi de langue et de culture espagnoles. Outre les cours de grammaire, il propose divers cours thématiques qui abordent des sujets d'intérêts variés par le biais de la langue espagnole. Il existe ainsi des cours de communication orale et de tourisme, d'histoire de l'Espagne et de l'Amérique latine et des sujets d'actualité dans les pays hispanophones, entre autres.

Initialement, nous avons observé que les étudiants des cours d'espagnol langue étrangère manquaient d'opportunités dans la pratique orale de l'espagnol en contexte naturel avec des locuteurs natifs. De fait, nous avons donc eu l'idée d'inviter en classe d'espagnol quelques étudiants natifs appartenant au programme UQAMMIDI, afin qu'ils puissent présenter leurs pays d'origine et partager l'information avec les étudiants francophones. Ce partage a donné naissance à un contact plus fréquent et plus équilibré entre les deux groupes de locuteurs. Les échanges interculturels ont ainisi vu le jour à partir du souhait d'enrichir la pratique orale des étudiants en cours d'espagnol langue étrangère et de ceux du programme de francisation du ministère de la même université. Les rencontres entre les hispanophones invités et les étudiants francophones nous ont motivés à aller plus loin et à offrir des séances de partage culturel, ou chaque étudiant francophone était jumelé à un étudiant hispanophone dans des activités planifiées et bien encadrées.

Comme nous croyons à la pérennité de ces activités et qu'un soutien institutionnel était important, des rencontres avec la direction de I'UQAM-MIDI et la direction du programme d'espagnol ont eu lieu. Ces démarches ont officialisé les jumelages linguistiques et interculturels dans les classes des deux programmes.

Ces activités, qui sont intégrées aux cours universitaires d'espagnol langue étrangère, ont trois objectifs principaux : (i) améliorer les pratiques actionnelles d'apprentissage (par le biais d'une activité qui a un but réel), (ii) promouvoir le respect mutuel des participants et (iii) valoriser l'Autre.

Une planification détaillée garantit la réussite du projet. Elle nécessite l'utilisation à la fois de ressources humaines et de matériel pédagogique. En ce qui concerne les ressources humaines, la coordinatrice des échanges linguistiques et interculturels est responsable de la création du matériel pédagogique en accord avec les objectifs et les niveaux linguistiques des cours impliqués dans les échanges. Elle est également responsable de la diffusion et du recrutement des étudiants hispanophones à ces rencontres.

Les enseignants d'espagnol, quant à eux, insèrent ces activités dans les plans de cours pour que la participation rentre dans les paramètres/critères d'évaluation de la matière. Pour ce qui est du matériel pédagogique, du contenu relié aux thématiques de chaque cours a été créé. Ce matériel complète les notions d'ordre plus théorique vues en classe.

Les bénéfices de cette activité s'appliquent aux deux groupes de participants. Pour les étudiants hispanophones, il constitue un moyen d'améliorer efficacement leurs compétences linguistiques en français ainsi que leur intégration scolaire ou professionnelle dans le nouveau contexte social où ils vivent. Pour les étudiants francophones, ces échanges permettent de mieux comprendre les composantes culturelles de différentes thématiques traitées en classe. Enfin, un dernier avantage soulevé pour les deux groupes d'étudiants est de dissiper les préjugés interculturels qui auraient pu être forgés lors de l'apprentissage de chacune des langues en question, que ce soit l'espagnol ou le français.

Du point de vue de la motivation, cette activité est très appréciée par les deux groupes de participants. La motivation a joué un facteur clé et a rendu possible la création d'une page Facebook encore active, où les participants partagent virtuellement des informations sur les événements culturels à Montréal, des sites de ressources linguistiques pour améliorer leurs connaissances dans les deux langues, ainsi que des opportunités d'étude ou de travail, tout en démontrant une solidarité et un climat de réseautage hors pair. L'engouement pour ces échanges va donc au-delà des murs des salles de classe de l'UQAM et tisse des liens très solides dans la communauté.

Un autre aspect très valorisé est le réseautage professionnel que ces activités offrent pour les étudiants hispanophones. En d'autres mots, l'organisation de jumelages dans le programme d'espagnol a comme objectif 
additionnel de consolider un espace d'échange et d'entraide pour les participants. En matière d'intégration économique, les participants collaborent pour l'intégration des hispanophones au monde du travail à Montréal. De leur côté, les étudiants francophones développent une curiosité qui va bien au-delà d'un cours d'espagnol, pour explorer davantage la réalité du monde hispanophone, que ce soit en participant dans des activités culturelles à Montréal ou en allant dans les pays d'origine de leurs jumeaux avec un nouveau regard de leur réalité.

Ces activités favorisent le rapprochement interculturel ainsi que l'intégration sociale, linguistique et culturelle des jumeaux immigrants et, en même temps, offrent une sensibilisation aux réalités de nouveaux arrivants pour les membres de la société d'accueil. 